\title{
EVOLUÇÃO DA PESQUISA EM RBV: um estudo dos últimos EnANPAD's
}

\section{The evolution of research on the RBV: a study of the latest ENANPAD'S}

\author{
Fernando A. Ribeiro Serra ${ }^{a}$, Manuel Portugal Ferreira ${ }^{\mathrm{b}}$, \\ Maurício Fernandes Pereira ${ }^{\mathrm{c}}$, Juliano Lissoni ${ }^{\mathrm{d}}$
}

\footnotetext{
${ }^{a}$ Engenheiro Metalúrgico, Dr. Prof. da Unisul Business School, Universidade do Sul de Santa Catarina. Florianópolis, SC - Brasil, e-mail: fernando.serra@unisul.br

${ }^{\mathrm{b}}$ Economista, Dr. Prof. da Escola Superior de Tecnologia e Gestão. Instituto Politécnico de Leiria Morro do Lena, Alto Vieiro Leiria - Portugal, e-mail: portugal@estg.ipleiria.pt

c Universidade Federal de Santa Catarina.Florianópolis, SC - Brasil.e-mail: mpereira@cse.ufsc.br

d Unisul Business School. Universidade do Sul de Santa Catarina. Florianópolis, SC - Brasil, e-mail: julianolissoni@gmail.com
}

\begin{abstract}
Resumo
O objetivo do presente trabalho foi verificar a contribuição brasileira nos estudos de estratégia e enfoque na RESOURCE-BASED VIEW - RBV. O estudo visou expor e analisar a evolução da pesquisa em RBV no Brasil, a partir dos trabalhos selecionados e apresentados no EnANPAD. No levantamento preliminar para a análise dos artigos, considerando 10 anos de EnANPAD (1997 - 2006), foram selecionados 57 artigos. A identificação e seleção dos artigos envolveram os seguintes critérios: avaliação dos títulos e resumos dos artigos apresentados na área de estratégia entre 1997 e 2006, busca complementar por palavras-chave tais como RBV, VBR, recursos, capacidades, competências, visão baseada em recursos e resource-based view, core competence e de dynamic capabilitiess na base de dados do EnANPAD. Procedemos também à análise das referências utilizadas nos artigos. Na primeira parte revemos brevemente aspectos fundamentais da RBV. Na segunda parte apresentamos a metodologia seguida na coleta dos dados e fazemos análise dos resultados. Concluímos com uma discussão geral propondo, a partir da evolução dos trabalhos nacionais, uma agenda que possa orientar pesquisas futuras.
\end{abstract}

Palavras-chave: RBV; Estratégia; EnANPAD.

\section{Abstract}

The main purpose of this work was to verify the Brazilian contribution in strategy studies in $R B V$. This study wishes to expose and analyses the $R B V$ research evolution in Brasil through the works presented at EnANPAD. A ten year period was considered, EnANPAD (1997- 2006). The selection of 57 articles with the following criteria: title and summary evaluation; search for 
complementary keywords as resource-based view, core competence and dynamic capabilities in EnANPAD data-base; use references in the individual articles. The first part of the article presents a short review of the fundamental issues of $R B V$. The second part presented the methodology followed from the data collection and results analysis. The conclusion, from a general discussion, proposes from the national works evolution a future research agenda.

Keywords: RBV; Strategy; EnANPAD.

\section{INTRODUÇÃO}

O estudo da estratégia se desenvolveu na tentativa em explicar e conduzir as organizações a conquistar vantagens competitivas sustentáveis. Nos últimos 50 anos, acadêmicos em todo o mundo têm teorizado sobre estratégia e, especificamente, sobre as fontes de sucesso das empresas (KAY, 1993; DeGEUS, 1997; DeGEUS, 1998; JOYCE et al., 2003). As teorias e conceitos desenvolvidos procuram explicar e compreender as diferenças de desempenho entre empresas. Diferentes correntes de pensamento emergem e aplicam-se as empresas, desde as baseadas na teoria evolucionária (NELSON; WINTER, 1982) e co-evolucionária (LEWIN et al., 1999), teoria dos custos de transação (COASE, 1937; WILLIAMSON, 1985), aprendizagem (LEVITT; MARCH, 1988), teoria da agência (ROSS, 1973), contigencial (BOYD, 1995), institucional (ZUCKER, 1987), das redes relacionais ou social networks (GRANOVETTER, 1985) e Resource-Based View - RBV (BARNEY, 1991). A academia mundial tem-se concentrado em abordagens mais orientadas para a empresa em vez de abordagens mais orientadas para a indústria onde a empresa opera (CAVES; PORTER, 1977; PORTER, 1980).

O estudo bibliométrico de RamosRodriguez e Ruiz-Navarro (2004), realizado nos artigos publicados no Strategic Management Journal, entre 1980 e 2000, concluiu a existência de um elevado potencial da abordagem teórica da RBV como uma possibilidade para responder ao desafio da busca pela vantagem competitiva sustentável.

O estudo da estratégia empresarial no Brasil tem se desenvolvido e tem sido influenciado significativamente pela literatura anglo-saxônica (BIGNETTI; PAIVA, 2001), fundamentalmente a originada nos Estados Unidos. No entanto, não dispomos ainda de uma noção exata da evolução dos estudos da estratégia no Brasil, em particular a focada na RBV.

Assim, a questão no presente estudo, parte de uma pesquisa mais abrangente que visa verificar a contribuição brasileira nos estudos de estratégia, e em particular o enfoque na RBV procurando responder a seguinte questão: Qual a evolução dos trabalhos científicos brasileiros em RBV?

O objetivo é, ao identificar a evolução e as áreas de pesquisa, possibilitar a proposição de uma agenda de pesquisa e verificar os possíveis colaboradores nessa agenda.

Neste artigo focamos em particular os trabalhos do EnANPAD, principal congresso da ANPAD - Associação Nacional de Pós-graduação e Pesquisa em Administração. Assim, na primeira parte revemos brevemente aspectos fundamentais da RBV e a agenda de pesquisa proposta por autores de renome internacional. $\mathrm{Na}$ segunda parte apresentamos a metodologia seguida na coleta dos dados e fazemos à análise dos resultados. Concluímos com uma discussão geral propondo, a partir da evolução dos trabalhos nacionais, uma agenda que possa orientar pesquisas futuras.

\section{REFERENCIAL TEÓRICO}

Os estudos de estratégia se desenvolveram na procura por explicar e encontrar uma forma de direcionar as organizações na busca pela vantagem competitiva (KAY, 1993). Apesar de não existir uma teoria única e exclusiva para a criação de estratégias empresariais, as organizações se servem de guias para facilitar a formulação e administração de seus ativos estratégicos (SERRA et al., 2002). As contribuições advêm de inúmeras fontes e correntes de pensamento, como já referido antes, mas tem havido um enfoque particular nos 
últimos anos numa corrente ainda emergente: a Resource-based View (RBV) (RAMOSRODRIGUEZ; RUIZ-NAVARRO, 2004). Ainda assim, permanece o desafio de responder a questões como: por que empresas alcançam sucesso ou falham? (PROENÇA, 1999). Segundo Kay et al. (2003) a RBV e o seu desenvolvimento continua a ser uma esperança neste sentido.

Explicar e compreender a estratégia e as fontes de vantagem competitiva sustentável é um anseio dos executivos e empreendedores. As empresas parecem ganhar e perder a sua posição competitiva na indústria, em virtude da sua própria atuação e da dos concorrentes. Por exemplo, uma porção significativa das empresas de grande porte que participaram do ranking da revista Exame Maiores e Melhores, em 1974, já estão fora da lista ou perderam o seu posto de liderança. Por outro lado, empresas novas como a Gol Transportes Aéreos, que se tornou a segunda empresa aérea mais lucrativa do mundo em cinco anos de existência, aparecem e desafiam as empresas instaladas. Também surgem no cenário mundial empresas oriundas de territórios e países antes considerados insuspeitos, como os países do leste da Europa, da América Latina e da Ásia.

Dada a ênfase à Resouce-Based View (RBV) (PETERAF, 1993), importa compreender sumariamente de que se trata. O trabalho de Penrose (1959) é reconhecido como a base da abordagem teórica da RBV (WERNERFELT, 1984; RAMOSRODRIGUEZ; RUIZ-NAVARRO, 2004). Penrose (1959) e outros autores (WERNERFELT, 1984; PETERAF, 1993) argumentam que o crescimento e sucesso das firmas dependem da posse (ainda que por posse não signifiquemos a propriedade, mas pelo menos o acesso, salvo situações específicas) e do uso efetivo de recursos heterogêneos e únicos ou de relacionamentos (RUMELT, 1984, 1987). Os recursos, por sua vez, para gerarem vantagem competitiva, precisam ser valiosos e raros, difíceis de imitar e de substituir (BARNEY, 1986, 1991).

Ao longo da década de 80 e no início da década de 90, foram construídas as bases da abordagem RBV com a contribuição de diversos autores como Lippman e Rumelt (1982) que desenvolveram os conceitos de ambigüidade causal; de Rumelt $(1984,1987)$ pelos seus trabalhos sobre o conceito de mecanismos de isolamento de recursos; de Dierickx e Cool (1989) que desenvolveram os conceitos de substituição, erosão de ativos e ambigüidade causal; de Ghemawat (1986) pelos seus trabalhos sobre conceito de inimitabilidade; de Castanias e Helfat (1991) pela discussão de qual o papel dos gerentes na geração das rendas; de Amit e Schoemaker (1993) pela sua contribuição para o conceito de vantagem competitiva sustentável a partir das imperfeições de recursos e das decisões gerenciais sobre o desenvolvimento e desdobramento de recursos; e de Hamel e Prahalad (1994) que trabalharam o conceito de core competence, ligado à RBV e comunicado em linguagem acessível aos executivos; entre vários outros.

Central à abordagem das RBV, surgem os recursos estratégicos que a empresa se deve esforçar por deter ou desenvolver. Os recursos são definidos por Wernerfelt (1984, p. 172) como "qualquer coisa que possa ser pensada com uma força ou fraqueza de uma dada firma. Mais formalmente, um recurso da firma, em dado tempo, pode ser definido como aqueles ativos (tangíveis e intangíveis) que são ligados semi-permanentemente à firma." Barney (1991, p. 101) também corrobora com o assunto ao afirmar que os recursos estratégicos "incluem todos os ativos, capacidades, processos organizacionais, atributos da firma, informação e conhecimento, [...] controlados por uma firma, que a habilitam a conceber e a implementar estratégias que melhorem sua eficiência e efetividade [...]. Na linguagem da análise estratégica tradicional, os recursos da firma são forças que as firmas podem conceber e implementar suas estratégias." Assim, os recursos precisam ser Valiosos para explorar as oportunidades e/ou neutralizar as ameaças no ambiente competitivo; Raros e não disponíveis a outros competidores; imperfeitamente imitáveis, ou Inimitáveis, na medida em que dependem de desenvolvimentos organizacionais específicos e são protegidos pela ambigüidade causal e complexidade social; e Não-substituíveis, na medida em que não existem recursos idênticos que permitam replicar um resultado semelhante (LIPPMAN; RUMELT, 1982; RUMELT, 1984; BARNEY, 1986; DIERICKX; COOL, 1989; BARNEY, 1991).

Destes trabalhos seminais o estudo da RBV evoluiu traçando fronteiras com outras abordagens teóricas. Mahoney (1995; 2001) e Mahoney e Pandian (1992) apresentam a idéia de complementaridade entre a RBV e outras teorias como aprendizagem organizacional e teoria dos 
custos de transação. Foss (1996), também argumenta sobre a complementaridade entre os aspectos contratuais - teoria dos custos de transação e direitos de propriedade - e os aspectos baseados em conhecimento - gestão do conhecimento e RBV. Hoopes, Madsen e Walker (2003) argumentam que a RBV fica clara quando vista como parte de uma teoria ampla de heterogeneidade competitiva combinando economia, teoria das organizações, política de negócios tradicional e RBV.

Adicionalmente, a partir da década de 90 diversos trabalhos empíricos procuraram suporte aos fundamentos observados pela RBV (MAHONEY, 2005).

\section{METODOLOGIA}

Este trabalho pretende avaliar as contribuições brasileiras para a evolução da abordagem teórica e aplicada da RBV. As fontes para este estudo podem ser inúmeras, como dissertações e teses, livros e artigos de congressos científicos e revistas científicas. Tal abrangência dificulta o trabalho em questão. No entanto, há conferências que são representativas dos trabalhos realizados no país. Sendo representativas, não são exclusivas, nem completamente inclusivas de toda a pesquisa realizada.

Assim, selecionamos uma fonte relevante para este estudo, no caso, o EnANPAD, principal congresso da ANPAD - Associação Nacional de Pós-Graduação e Pesquisa em Administração, organização que representa os principais programas de Pós-Graduação em Administração do Brasil e que organiza os principais e mais referenciados congressos ligados à administração, contemplando estudos e investigação na área da estratégia empresarial. O EnANPAD conta atualmente com onze áreas acadêmicas ADI - Administração da Informação, APS - Administração Pública e Gestão Social, ESO - Estratégia em Organizações, EOR Estudos Organizacionais, FIN - Finanças, GCT Gestão de Ciência, Tecnologia e Inovação, GPR Gestão de Pessoas e Relações de Trabalho, GOL Gestão de Operações e Logística, MKT - Marketing, EPQ - Ensino e Pesquisa em Administração e Contabilidade, CON - Contabilidade. Cada área tem ainda sub-áreas, que no caso de Estratégia em Organizações são as seguintes: ESO-A - Estratégia em Organizações, ESO-B - Gestão Internacional, ESO-C - Empreendedorismo e Comportamento Empreendedor.

Vergara (1997) propõe que os tipos de pesquisa sejam classificados genericamente quanto aos fins e quanto aos meios. Quanto aos fins, este estudo é descritivo, pois visa expor a evolução da pesquisa em RBV no Brasil, a partir dos trabalhos selecionados e apresentados no EnANPAD. É também explicativo ao pretender analisar a evolução da produção acadêmica dos trabalhos de estratégia na abordagem RBV.

Quanto aos meios, selecionamos como referido, para a pesquisa bibliográfica, apenas os trabalhos em RBV, ou com esta diretamente relacionados, apresentados no EnANPAD nos últimos dez anos. Todas as referências nacionais selecionadas foram analisadas quanto ao conteúdo e classificadas para exame.

Nolevantamento preliminar considerando 10 anos de EnANPAD, de 1997 até 2006, foram selecionados 57 artigos, conforme Tabela 1. A identificação e seleção dos artigos envolveram os seguintes critérios: avaliação dos títulos e resumos dos artigos apresentados na área de estratégia entre 1997 e 2006; busca complementar por palavraschave tais como RBV, VBR, recursos, capacidades, competências, visão baseada em recursos e resourcebased view, core competence (ou competência central) e de dynamic capabilitiess na base de dados do EnANPAD. Procedemos também à análise das referências utilizadas nos artigos. Isto é, analisamos os artigos verificando se um conjunto de palavraschave se encontrava nos campos referidos.

É interessante notar que a RBV só passa a ter a atenção dos pesquisadores que freqüentaram o EnANPAD em 1999. Isto é, só cerca de quinze anos depois do trabalho seminal de Wernerfelt (1984) e oito anos depois do trabalho fundamental de Barney (1991). No entanto, é, sobretudo a partir de 2001, que os trabalhos de pesquisadores brasileiros passaram a apresentar uma quantidade razoável e significativa de trabalhos em relação ao total de trabalhos na área de estratégia do EnANPAD (ver Tabela 1). 
TABELA 1 - Dados preliminares sobre os trabalhos selecionados

\begin{tabular}{|c|c|c|c|c|c|c|c|}
\hline Ano & $\mathbf{R B V}$ & Estrat. & $\% \mathrm{RBV}$ & RBV Total & N Autores & $\begin{array}{l}\text { Média } \\
\text { autores }\end{array}$ & $\begin{array}{c}\text { Tipo de } \\
\text { artigo (1) }\end{array}$ \\
\hline 2006 & 10 & 60 & 16,7 & 10 & 21 & 2,1 & $\mathrm{~T}(0), \mathrm{E}(8), \mathrm{C}(2)$ \\
\hline 2005 & 13 & 70 & 20,0 & 13 & 32 & 2,3 & $\mathrm{~T}(1), \mathrm{E}(10), \mathrm{C}(2)$ \\
\hline 2004 & 9 & 50 & 18,0 & 12 & 27 & 2,3 & $\mathrm{~T}(1), \mathrm{E}(8), \mathrm{C}(3)$ \\
\hline 2003 & 7 & 49 & 14,3 & 7 & 17 & 2,4 & $\mathrm{~T}(1), \mathrm{E}(1), \mathrm{C}(5)$ \\
\hline 2002 & 3 & 55 & 5,5 & 3 & 7 & 2,3 & $\mathrm{~T}(0), \mathrm{E}(1), \mathrm{C}(2)$ \\
\hline 2001 & 9 & 57 & 15,8 & 9 & 15 & 1,7 & $\mathrm{~T}(2), \mathrm{E}(3), \mathrm{C}(4)$ \\
\hline 2000 & 2 & 39 & 5,1 & 2 & 3 & 1,5 & $\mathrm{~T}(0), \mathrm{E}(0), \mathrm{C}(2)$ \\
\hline 1999 & 1 & 30 & 3,3 & 1 & 3 & 3,0 & $\mathrm{~T}(1), \mathrm{E}(0), \mathrm{C}(0)$ \\
\hline 1998 & 0 & 31 & 0 & - & - & - & - \\
\hline 1997 & 0 & 28 & 0 & - & - & - & - \\
\hline Total & 54 & - & - & 57 & 125 & - & - \\
\hline
\end{tabular}

Fonte: Elaborado pelos autores.

Nota: (0) tipo de artigo: T - Teórico, E - Empírico, C - Caso de estudo.

RBV - quantidade de artigos de RBV na área acadêmica de estratégia no ENANPAD;

Estratégia - quantidade total de artigos na área acadêmica de estratégia (atualmente ESO A);

$\%$ RBV - percentual de artigos em RBV em relação ao total do track de estratégia;

RBV total - considera a soma a quantidade anterior de artigos em RBV apresentados em outras áreas do EnANPAD;

$\mathrm{N}$ autores - quantidade total de autores dos artigos RBV no ano;

Média autores - média de autores por artigo no ano ( $\mathrm{N}$ autores / RBV);

Do total de 57 artigos selecionados e relacionados à RBV, 54 são da divisão acadêmica de Estratégia em Organizações (sub-área ESOA) e 3 de outras divisões acadêmicas (Estudos Organizacionais e Marketing).

A seleção do tipo de artigo em casos de estudo, empíricos e teóricos, foi feita pela leitura da metodologia e avaliação de cada um dos artigos selecionados.

É interessante notar que o estudo de casos continua a ser marcante desde o início, embora exista um peso aparentemente crescente dos artigos empíricos. Em 2006, por exemplo, dos 10 artigos identificados, 8 foram estudos empíricos, 2 estudos de caso e nenhum apenas teórico. $\mathrm{O}$ estudo de casos parece ter perdido predomínio. Interessante também notar os esforços colaborativos que se têm mantido já, que a média do número de autores se situa consistentemente em torno de dois, numa tendência ascendente.

Por fim, é importante salientar que os artigos utilizados como objeto da pesquisa não estão listados nas referências do artigo em tela, porém podemos encontrá-los nos quadros.

\section{RESULTADOS}

Uma análise mais detalhada pode elucidar a evolução específica e concentração de autores dominantes e, porventura, mais influentes nesta área do conhecimento de estratégia empresarial. Verificando os trabalhos selecionados, alguns autores apresentaram mais de uma vez nos EnANPAD ao longo dos dez anos, na área da RBV. Estes são: Pascucci e Grave, em 2003 e 2006; Almeida, em 2005 e 2006; Kayo e Basso, em 2006; Dias, em 2004 e 2006; Thomaz, Lerner e Brito, em 2004, 2005 e 2006; Hexsel, em 2003; Bitencourt, 2004, 2005 e 2006 apud Baraniuk e Bulgacov, em 2002 e 2003; Fensterseifer e Wilk em 2003; Kohls e Fensterseifer em 2004; Wilk em 2000 (Quadro 1). 
QUADRO 1 - Autores que publicam mais de uma vez no EnANPAD em RBV.

\begin{tabular}{|c|c|c|c|}
\hline Autores & Título & EnANPAD & Tipo de artigo \\
\hline Pascucci, L., & Ambiente institucional, recursos e comportamento & \multirow[t]{3}{*}{2006} & \multirow[t]{3}{*}{ Empírico } \\
\hline Crubellate, J.e & estratégico: $\mathrm{O}$ estudo da verticalização de negócios & & \\
\hline Grave, P. & em uma Unidade Regional da UNIMED. & & \\
\hline Pascucci, L. Grave, & A Unimed Maringá e seu posicionamento estratégico: & \multirow[t]{2}{*}{2003} & \multirow[t]{2}{*}{ Estudo de caso } \\
\hline P. e Silveira, J. & Uma explicação a partir da Visão Baseada em Recursos & & \\
\hline $\begin{array}{l}\text { Almeida A., Bertucci, } \\
\text { J. e Belo, J. }\end{array}$ & $\begin{array}{l}\text { A influência da comunicação na identificação dos } \\
\text { empregados com a organização e sua valorização } \\
\text { como recurso estratégico. }\end{array}$ & 2006 & Empírico \\
\hline Almeida, A. e & A construção da reputação organizacional como recurso & \multirow[t]{2}{*}{2005} & \multirow[t]{2}{*}{ Empírico } \\
\hline Muniz, R. & estratégico: O papel dos gestores e a percepção dos stakeholders. & & \\
\hline $\begin{array}{l}\text { Kayo, E., Basso, F. e } \\
\text { Penner-Hahn, J. }\end{array}$ & $\begin{array}{l}\text { The value relevance of intangible capabilities de } \\
\text { plovment: The role of firm life cycle. }\end{array}$ & 2006 & Empírico \\
\hline Meirelles, D., Basso, & Um estudo dos relacionamentos externos da empresa: & \multirow[t]{2}{*}{2005} & \multirow{2}{*}{ Empírico } \\
\hline L. e Pace, E.. & $\begin{array}{l}\text { Uma alicação da Teoria Baseada em Recursos ao Setor } \\
\text { de Software Brasileiro. }\end{array}$ & & \\
\hline Dias, A. & $\begin{array}{l}\text { Relações entre tamanho, diversificação, gestão de recursos, } \\
\text { participação de mercado e desempenho organizacional de } \\
\text { companhias brasileiras de capital aberto. }\end{array}$ & 2006 & Empírico \\
\hline $\begin{array}{l}\text { Dias, A., Gonçalves, } \\
\text { C. e Coleta, K. }\end{array}$ & $\begin{array}{l}\text { Determinantes estratégicos do desempenho de empresas em } \\
\text { ambientes turbulentos. }\end{array}$ & 2004 & Empírico. \\
\hline $\begin{array}{l}\text { Thomaz, J., Lerner, } \\
\text { E. e Brito, E. }\end{array}$ & $\begin{array}{l}\text { Reputação corporativa: Desenvolvendo uma escala } \\
\text { de mensuração. }\end{array}$ & 2006 & Empírico \\
\hline Brito, E., Campos, L., & Reputação corporativa e desempenho: Uma análise & \multirow[t]{2}{*}{2005} & \multirow[t]{2}{*}{ Empírico } \\
\hline Brito, L. e Thomaz, J. & empírica no setor bancário. & & \\
\hline $\begin{array}{l}\text { Brito, E., Thomaz, } \\
\text { J. e Brito, L. }\end{array}$ & Corporate reputation as a strategic resource. & 2004 & Empírico \\
\hline Santos, M. e & A obtenção da vantagem competitiva através do & \multirow[t]{2}{*}{2005} & \multirow{2}{*}{ Estudo de Caso } \\
\hline Hexsel, A. & $\begin{array}{l}\text { desenvolvimento de competências organizacionais } \\
\text { a partir da combinação e integração de recursos. }\end{array}$ & & \\
\hline Hexsel, A. & $\begin{array}{l}\text { O entendimento do sucesso internacional da Marcopolo: } \\
\text { Uma visão com base em recursos. }\end{array}$ & 2003 & Estudo de Caso \\
\hline Dolabela, R. e & A consolidação das competências organizacionais na & \multirow[t]{2}{*}{2006} & \multirow[t]{2}{*}{ Estudo de caso } \\
\hline Bitencourt, C. & vitivinicultura brasileira: Um estudo de caso da vinícola Miolo. & & \\
\hline Tondolo, V. e & Uma perspectiva baseada em recursos no agronegócio & \multirow[t]{2}{*}{2005} & \multirow[t]{2}{*}{ Estudo de caso } \\
\hline Bitencourt, C. & cooperativo: O caso CCGL. & & \\
\hline Bitencourt, C. e & A consolidação de competências com base em práticas & \multirow[t]{2}{*}{2004} & \multirow[t]{2}{*}{ Estudo de caso } \\
\hline Gonçalo, C. & informais: Uma opção estratégica na ação organizacional. & & \\
\hline Hayashi Jr, P., & Recursos e competências nas mudanças de conteúdo & \multirow[t]{3}{*}{2003} & \multirow[t]{3}{*}{ Estudo de caso } \\
\hline Baraniuk, J. e & estratégico em pequenas empresas de massas alimentícias: & & \\
\hline Bulgacov, S. & Estudo comparativo de casos. & & \\
\hline Baraniuk, J. e & Conteúdo e recursos estratégicos de empresas novas entrantes & 2002 & Estudo de caso \\
\hline Bulgacov, $\mathrm{S}$, & provedoras de serviços de comunicação de dados do Paraná. & & \\
\hline Fensterseifer, J. e & Visão da firma baseada em recursos, clusters e performance: & 2005 & Empírico \\
\hline Wilk, E. & Um estudo no setor vitivinícula do RS. & & \\
\hline Kohls, V. e & Estratégias competitivas de empresas agroalimentares: & 2004 & Estudo de caso \\
\hline Fensterseifer, J. & Estudos de casos na zona sul do RS. & & \\
\hline Wilk, E. e & Alianças estratégicas sob a perspectiva da visão da firma & 2003 & Estudo de caso \\
\hline Fensterseifer, J. & $\begin{array}{l}\text { baseada em recursos: Contribuições para um modelo } \\
\text { dinâmico de cooperação. }\end{array}$ & & \\
\hline Wilk, E. & $\begin{array}{l}\text { O uso de uma abordagem Resource Based View na } \\
\text { formulação de estratégias: O caso do setor vitivinícula } \\
\text { da Serra Gaúcha. }\end{array}$ & 2000 & Estudo de caso \\
\hline
\end{tabular}

Fonte: Análise dos autores.

Obs.: O quadro está organizado de forma a apresentar os autores que publicam mais de uma vez ao longo dos dez anos do ENANPAD em RBV, em conjunto com quem trabalham. Não considera ordem 
Observando o currículo Lattes dos autores mencionados, como disponibilizado no site do CNPq (www.cnpq.br) verificamos que Fensterseifer, Wilk e Basso pesquisam utilizando a abordagem RBV, Bitencourt em Competências, Hexsel estratégia em geral e Baraniuk e Bulgacov pesquisam conteúdo e processo estratégico, relacionados com a RBV. Os demais autores não aparentam um foco tão evidente e, talvez, tenham-se utilizado da RBV para suportar as avaliações em seus objetos de pesquisa (ver, por exemplo, os trabalhos de Almeida ou do grupo Thomaz, Brito e Brito sobre "reputação"). É notório, no entanto, pela leitura do Quadro 1 a importância do estudo de caso no desenvolvimento da RBV.

Dado que o enfoque da RBV está nos recursos, competências ou dynamic capabilities que a empresa dispõe ou a que tem acesso, importa verificar quais são os tipos de recursos sobre que tem incidido a pesquisa brasileira (Quadro 2). Recordemos este propósito, que Barney (1991) avançou, de que os recursos estratégicos podem incluir uma ampla variedade de aspectos, tais como: capacidades, processos organizacionais, atributos da firma, informação e conhecimento.

QUADRO 2 - Autores e recursos estratégicos focados

\begin{tabular}{|c|c|c|c|}
\hline Autores & Título do artigo & Ano & $\begin{array}{l}\text { Tipo de recurso } \\
\text { estratégico }\end{array}$ \\
\hline Jacques, J. & $\begin{array}{l}\text { A importância da análise do contexto organizacional durante } \\
\text { a construção e implementação de recursos assistenciais na gestão } \\
\text { da criação do conhecimento uma contribuição para o processo } \\
\text { estratégico nas organizações hospitalares. }\end{array}$ & 2006 & $\begin{array}{l}\text { Protocolos médico- } \\
\text { assistênciais }\end{array}$ \\
\hline $\begin{array}{l}\text { Almeida, A., Bertucci, J. } \\
\text { e belo, J. }\end{array}$ & $\begin{array}{l}\text { A influência da comunicação na identificação dos empregados } \\
\text { com a organização e sua valorização como recurso estratégico. }\end{array}$ & 2006 & Comunicação interna \\
\hline $\begin{array}{l}\text { Thomaz, J, Lerner, E. e } \\
\text { Brito, E. }\end{array}$ & Reputação corporativa desenvolvendo uma escala de mesuração. & 2006 & Reputação corporativa \\
\hline $\begin{array}{l}\text { Brito, E, Campos, L. e } \\
\text { Thomaz, J. }\end{array}$ & $\begin{array}{l}\text { Reputação corporativa e desempenho: uma análise empírica } \\
\text { no setor bancário. }\end{array}$ & 2005 & Reputação corporativa \\
\hline Almeida, A. e Muniz, R. & $\begin{array}{l}\text { A construção de reputação organizacional como recurso } \\
\text { estratégico: o papel dos gestores e a percepção dos stakeholders. }\end{array}$ & 2005 & Reputação corporativa \\
\hline Omaki, E. & $\begin{array}{l}\text { Recursos intangíveis e desempenho em grandes empresas } \\
\text { brasileiras: avaliações dos recursos intangíveis como estimadores } \\
\text { de medidas de desempenho financeiras. }\end{array}$ & 2005 & Reputação corporativa \\
\hline Pires, M. e Marcondes, R. & $\begin{array}{l}\text { A vantagem competitiva nas organizações financeiras: uma } \\
\text { análise baseada na Teoria dos Recursos. }\end{array}$ & 2004 & $\begin{array}{l}\text { Tecnologia da informação } \\
\text { e do conhecimento }\end{array}$ \\
\hline Hofmann, V. & $\begin{array}{l}\text { La ventaja competitiva basada em la estratégia de } \\
\text { perfeccionamiento operacional de los recursos humanos: } \\
\text { el caso de uma red de supemercados em Brasil. }\end{array}$ & 2001 & Recursos humanos \\
\hline
\end{tabular}

Fonte: Análise dos autores. 
Uma porção significativa dos trabalhos procurou identificar ou justificar determinados recursos como recursos estratégicos importantes para conquistar vantagem competitiva sustentável (Quadro 2). Jacques (2006) avaliou os protocolos médico-assistenciais como instrumentos estratégicos; e Almeida, Bertucci e Belo (2006) o processo de comunicação interna como um recurso fundamental para a empresa; Thomaz, Lerner e Brito (2006); Brito et al. (2005); Almeida e Muniz (2005); Omaki (2005) avaliam a reputação corporativa como fonte de vantagem competitiva; Pires e Marcondes (2004) focam a importância das tecnologias da informação e do conhecimento; Hofmann (2002) investigou os recursos humanos como fonte de vantagem competitiva a partir do estudo de caso de uma rede de supermercados brasileira.

Além dos aspectos já referidos, diversos trabalhos ao longo destes dez anos de EnANPAD procuraram explicar a vantagem competitiva de empresas individuais e de setores de atividade. Exemplos destes trabalhos são apresentados no Quadro 3:

QUADRO 3 - Autores e alguns exemplos de trabalhos de indústrias e empresas estudadas

\begin{tabular}{|c|c|c|c|}
\hline Autores & Título do artigo & Ano & $\begin{array}{l}\text { Tipo de recurso } \\
\text { estratégico }\end{array}$ \\
\hline Silva , J. e Oliveira, C. & $\begin{array}{l}\text { Grupos estratégicos e a visão resource-based view: } \\
\text { Uma aplicação de sistemas Neuro-fuzzy. }\end{array}$ & 2005 & Supermercados \\
\hline $\begin{array}{l}\text { Meirelles, D., Basso, } \\
\text { L. e Pace, E. }\end{array}$ & $\begin{array}{l}\text { Um estudo dos relacionamentos externos da empresa: } \\
\text { Uma aplicação da teoria baseada em recursos ao setor de software }\end{array}$ & $\begin{array}{l}2005 \\
\text { orasileiro. }\end{array}$ & Setor de Software \\
\hline $\begin{array}{l}\text { Sousa, J., Ferreira, F. } \\
\text { e Moura, S. }\end{array}$ & $\begin{array}{l}\text { Proposta de categorização dos recursos estratégicos de IES } \\
\text { particular: Uma contribuição com base nos modelos da } \\
\text { VBR e da Hipercompetição. }\end{array}$ & 2005 & $\begin{array}{l}\text { Inst. Ensino Superior } \\
\text { (IES) }\end{array}$ \\
\hline $\begin{array}{l}\text { Fensterseifer, J. e } \\
\text { Wilk, E. }\end{array}$ & $\begin{array}{l}\text { Visão da firma baseada em recursos, clusters e performance: } \\
\text { Um estudo do setor vitivinícola do RS. }\end{array}$ & 2005 & Cluster \\
\hline Pereira, $\mathrm{M}$. & $\begin{array}{l}\text { Visão baseada em recursos nas Instituições de Ensino } \\
\text { Superior de Fortaleza: Uma análise Ex-ante e Ex-post à LDB/96. }\end{array}$ & 2004 & IES \\
\hline $\begin{array}{l}\text { Pascucci, L., Grave, } \\
\text { P. e Silveira, J. }\end{array}$ & $\begin{array}{l}\text { A Unimed Maringá e seu posicionamento estratégico: } \\
\text { Uma explicação a partir da Visão Baseada em Recursos. }\end{array}$ & 2003 & Org. saúde (Unimed) \\
\hline Hexsel, A. & $\begin{array}{l}\text { O entendimento do sucesso internacional da Marcopolo: } \\
\text { Uma visão com base em recursos. }\end{array}$ & 2003 & Montadora (Marcopolo) \\
\hline Wilk, E. e Fensterseifer, J. & $\begin{array}{l}\text { Alianças estratégicas sob a perspectiva da visão da firma } \\
\text { baseada em recursos: Contribuições para um modelo } \\
\text { dinâmico de cooperação. }\end{array}$ & 2003 & Montadora (Marcopolo) \\
\hline Binder, $\mathrm{M}$. & $\begin{array}{l}\text { Rede de recursos: Um modelo desenvolvido a partir do caso } \\
\text { Gol Linhas Aéreas. }\end{array}$ & 2003 & Trnasp. Aéreo (GOL) \\
\hline Rosário, F. e Barboza, J. & Recursos e posicionamento competitivo nas PME's. & 2002 & PME (óticas) \\
\hline Baraniuk, J. e Bulgacov, S. & $\begin{array}{l}\text { Conteúdo e recursos estratégicos de empresas novas entrantes } \\
\text { provedoras de serviços de comunicação de dados no Paraná. }\end{array}$ & 2002 & Comunicação de dados \\
\hline Ferreira, P. e Mariano, S. & $\begin{array}{l}\text { Estratégia competitiva no mundo virtual: } \\
\text { O caso Americanas.com. }\end{array}$ & 2001 & E-commerce \\
\hline
\end{tabular}

Fonte: Análise dos autores. 
A análise das indústrias e empresas estudadas permite-nos observar grande diversidade em termos do objeto de estudo que é possível abarcar usando a RBV. Alguns autores focaram o estudo de setores de atividade, como por exemplo, Silva e Oliveira (2005) com os supermercados, Meirelles, Basso e Pace (2005) o setor do software, para o qual integrou a importância dos relacionamentos externos e os recursos da empresa; Baraniuk e Bulgacov (2002) investigam o conteúdo e recursos estratégicos utilizados por novas entrantes no nicho de comunicação de dados no estado do Paraná. Outros focaram organizações específicas, como Sousa, Ferreira e Moura. (2005) procuraram caracterizar os recursos de uma IES particular, Pereira (2004) que procura identificar os recursos relevantes antes e após a LDB/96, Pascucci et al. (2003) procuram explicar o posicionamento da Unimed; Hexsel (2003) apresenta uma avaliação da estratégia internacional da Marcopolo; Ferreira e Mariano (2002) avaliam a estratégia competitiva da Americanas.com e Binder (2003) que apresenta uma análise dos recursos que geram vantagem competitiva no caso da Gol Transportes Aéreos. Ainda outros autores focam sistemas e atuações mais complexas, como o estudo de Andrade (2006) sobre a gestão das universidades; Wilk e Fensterseifer (2003) usam o caso da Marcopolo para explicar as alianças estratégicas no processo de internacionalização da empresa;

Somente três trabalhos teóricos foram verificados (Quadro 4): Carneiro et al. (1999) - o primeiro identificado sobre RBV e apresentado no EnANPAD, neste trabalho os autores revisaram a literatura sobre RBV e procuraram passar uma perspectiva para os pesquisadores $\mathrm{e}$ executivos brasileiros numa alternativa à análise das forças da indústria, (PORTER, 1980), dominante nas escolas e universidades; Bandeira de Mello e Cunha (2001) - propuseram uma agenda para pesquisas sobre vantagem competitiva das empresas brasileiras com base na natureza e dinâmica das capacidades organizacionais; Kimura, Martin e Nakamura (2004) apresentaram modelo matemático procurando integrar capability building e resource picking para a obtenção de economic rents.

QUADRO 4 - Trabalhos teóricos sobre RBV no ENANPAD

\begin{tabular}{|c|c|c|c|}
\hline Autores & Título do artigo & Ano & Aspecto Teórico \\
\hline $\begin{array}{l}\text { Carneiro, J., Cavalcanti, } \\
\text { M. A., Silva, J. }\end{array}$ & $\begin{array}{l}\text { Os determinantes da sustentabilidade da Vantagem Competitiva } \\
\text { na Visão Rescurce-based. }\end{array}$ & 1999 & $\begin{array}{l}\text { Revisão da literatura } \\
\text { e proposta de agenda } \\
\text { de pesquisa em RBV. }\end{array}$ \\
\hline $\begin{array}{l}\text { Bandeira de Mello, R, } \\
\text { Cunha, C. }\end{array}$ & $\begin{array}{l}\text { A natureza e a Dinâmica das Capacidades Organizacionais no } \\
\text { Contexto Brasileiro: Uma agenda para Pesquisas sobre a } \\
\text { Vantagem Competitiva das Empresas Brasileiras. }\end{array}$ & 2001 & $\begin{array}{l}\text { Revisão da literatura } \\
\text { de capacidades dinâmicas } \\
\text { e proposta de agenda } \\
\text { para pesquisa. }\end{array}$ \\
\hline $\begin{array}{l}\text { Kimura H., Martin, D., } \\
\text { Nakamura, W. }\end{array}$ & $\begin{array}{l}\text { Modelagem de Economic Rents através das Vantagens de } \\
\text { Capability-Building e de Resource-Picking. }\end{array}$ & 2004 & $\begin{array}{l}\text { Proposta de modelo } \\
\text { matemático integrando } \\
\text { aspectos de RBV e } \\
\text { capacidades. }\end{array}$ \\
\hline
\end{tabular}

Fonte: Análise dos autores.

O conceito de competência apresentado por Prahalad e Hamel (1990) influenciou bastante a produção acadêmica nacional nos EnANPAD. Segundo Dolabella e Bitencourt (2006) num estudo sobre as competências organiza-cionais da vinícola Miolo a "discussão sobre as competências organizacionais tem sua origem na teoria da Visão Baseada em Recursos...”
Os estudos de competências, em geral, procuraram relacionar-se com a RBV, sendo o recurso antecessor à competência. Os trabalhos ligados a competências, de forma análoga aos diretamente ligados à RBV, procuraram identificar ou justificar a vantagem competitiva a partir das competências (Quadro 5). 
QUADRO 5 - Trabalhos ligados a competências

\begin{tabular}{|c|c|c|c|}
\hline Autores & Título do artigo & Ano & Aspecto Teórico \\
\hline Assumpção, J. & $\begin{array}{l}\text { Papel das competências técnico-organizacionais na estratégia } \\
\text { organizacional: Evidências de cinco organizações } \\
\text { não-governamentais no Rio de Janeiro. }\end{array}$ & 2006 & $\begin{array}{l}\text { Estudou a influência das } \\
\text { competências técnico- } \\
\text { organizacionais na } \\
\text { estratégia. }\end{array}$ \\
\hline $\begin{array}{l}\text { Carvalho, S. e } \\
\text { Barboza, A. }\end{array}$ & $\begin{array}{l}\text { O desenvolvimento de competências organizacionais: Um } \\
\text { estudo aplicado ao ensino a distância via Internet. }\end{array}$ & 2005 & $\begin{array}{l}\text { Descreveram e analisaram } \\
\text { o desenvolvimento } \\
\text { de competências } \\
\text { organizacionais }\end{array}$ \\
\hline Becker, G. e Ruas, R. & $\begin{array}{l}\text { Formação e desenvolvimento de competências organizacionais: } \\
\text { desvendando uma trajetória na competitividade empresarial. }\end{array}$ & 2005 & $\begin{array}{l}\text { Identificação e } \\
\text { mensuração de } \\
\text { competências } \\
\text { organizacionais. }\end{array}$ \\
\hline Correa, D. & $\begin{array}{l}\text { Estratégias para a construção de competências coletivas no } \\
\text { esporte: O caso do futebol profissional. }\end{array}$ & 2005 & $\begin{array}{l}\text { Importância da gestão } \\
\text { por competências. }\end{array}$ \\
\hline Santos, M. e Hexsel, A. & $\begin{array}{l}\text { A obtenção de vantagem competitiva através do } \\
\text { desenvolvimento de competências organizacionais a partir } \\
\text { da combinação e integração de recursos. }\end{array}$ & 2005 & $\begin{array}{l}\text { Metodologia para } \\
\text { identificação e } \\
\text { mensuração das } \\
\text { competências. }\end{array}$ \\
\hline $\begin{array}{l}\text { Contador, J. L., Ryllo, } \\
\text { E. e Contador, J. C. }\end{array}$ & Determinação do core business da empresa. & 2004 & $\begin{array}{l}\text { Metodologia } \\
\text { quantitativa para } \\
\text { identificação de core } \\
\text { business e core competence. }\end{array}$ \\
\hline Gomes, C., Gouvêa, & $\begin{array}{l}\text { Avaliação da competência estratégica nas organizações. } \\
\text { M. e Amorim, W. }\end{array}$ & 2004 & $\begin{array}{l}\text { Avaliou a competência } \\
\text { estratégica do Sindicato } \\
\text { dos Trabalhadores das } \\
\text { Indústrias Metalúrgicas, } \\
\text { Mecânicas e do Material } \\
\text { Elétrico de Osasco e } \\
\text { Região (STIMME). }\end{array}$ \\
\hline Bellinghini, $\mathrm{M}$. & $\begin{array}{l}\text { Estratégias de acumulação de competências tecnológicas e } \\
\text { aprimoramento de performance técnico-econômica em operadoras } \\
\text { de telefonia fixa: A experiência da Telemar Norte Leste SA. }\end{array}$ & 2004 & $\begin{array}{l}\text { Investigaram a } \\
\text { acumulação de } \\
\text { competências para o } \\
\text { desenvolvimento de } \\
\text { produtos, impantação } \\
\text { de sistemas e processos. }\end{array}$ \\
\hline $\begin{array}{l}\text { Bitencourt, C. e } \\
\text { Gonçalo, C. }\end{array}$ & $\begin{array}{l}\text { A consolidação de competências com base nas práticas informais: } \\
\text { Uma opção estratégica na ação organizacional. }\end{array}$ & 2004 & $\begin{array}{l}\text { Consolidação de } \\
\text { competências a partir de } \\
\text { práticas informais. }\end{array}$ \\
\hline
\end{tabular}




\begin{tabular}{|c|c|c|c|}
\hline $\begin{array}{l}\text { Hayashi Jr, P., Baraniuk, } \\
\text { J. e Bulgacov, S. }\end{array}$ & $\begin{array}{l}\text { Recursos e competências nas mudanças de conteúdo estratégico } \\
\text { em pequenas empresas de massas alimentícias: Estudo comparativ } \\
\text { de casos. }\end{array}$ & 2003 & $\begin{array}{l}\text { Estudaram recursos } \\
\text { relevantes e seus } \\
\text { impactos sobre a } \\
\text { vantagem competitiva } \\
\text { de três empresas de } \\
\text { pequeno porte. }\end{array}$ \\
\hline $\begin{array}{l}\text { Büttenbender, P. e } \\
\text { Figueiredo, P. }\end{array}$ & $\begin{array}{l}\text { Acumulação de competências tecnológicas e os processos } \\
\text { subjacentes de aprendizagem na indústria metal-mecânica: } \\
\text { O caso da AGCO - indústria de colheitadeiras. }\end{array}$ & 2002 & $\begin{array}{l}\text { Estudaram a } \\
\text { acumulação de } \\
\text { competências } \\
\text { tecnológicas e os } \\
\text { processos numa } \\
\text { indústria metal-mecânica } \\
\text { de pequeno/médio } \\
\text { porte. }\end{array}$ \\
\hline Harb, A. & $\begin{array}{l}\text { A determinação das competências essenciais: O caso no setor } \\
\text { supermercadista da cidade de Manaus. }\end{array}$ & 2001 & $\begin{array}{l}\text { Identificaram as } \\
\text { competências essenciais, } \\
\text { segundo a percepção de } \\
\text { gestores, funcionários e } \\
\text { clientes em empresas do } \\
\text { setor supermercadista. }\end{array}$ \\
\hline $\begin{array}{l}\text { Marques, D. e } \\
\text { Moraes, W. }\end{array}$ & $\begin{array}{l}\text { Desempenho competitivo: Capacidades diferenciadoras } \\
\text { e posicionamento competitivo. }\end{array}$ & 2001 & $\begin{array}{l}\text { Apresentaram a relação } \\
\text { entre competências } \\
\text { essenciais e estratégias } \\
\text { empresariais baseadas } \\
\text { no conhecimento. }\end{array}$ \\
\hline
\end{tabular}

Fonte: Análise dos autores.

\section{DISCUSSÃO DOS RESULTADOS}

A análise dos textos apresentados no EnANPAD permite-nos aferir alguns pontos sobre a evolução dos estudos de estratégia numa óptica de RBV. É evidente o peso relativo dos casos de estudo, com um crescimento relativo dos artigos empíricos em relação aos artigos puramente teóricos, ao longo dos últimos dez anos. Aqui aparece uma primeira oportunidade e recomendação para uma agenda futura de pesquisa em RBV. Existe espaço para artigos teóricos, tanto nas abordagens ligadas à RBV, como competência central, capacidades dinâmicas e gestão do conhecimento, quanto em trabalhos que envolvam a RBV e sua ligação com outras abordagens teóricas.

De acordo com os dados levantados, nos parece adequado apresentar uma proposta de agenda de pesquisas futuras para a RBV. Além disso, para os próprios autores este exercício será importante para direcionar suas pesquisas futuras de forma a, com o tempo, obter resultados que venham a agregar na geração de conhecimento sobre RBV e suas derivações.

Mahoney (2005) avaliou as idéias centrais de cinco abordagens da economia organizacional: a teoria comportamental da firma, a teoria dos custos de transação (TCT), a teoria dos direitos de propriedade, a teoria da agência e a RBV, em conjunto com capacidades dinâmicas e opções reais. A partir daí sugeriu diversas questões de pesquisa. Antes de apresentar algumas destas questões selecionadas, a própria interface com estas abordagens teóricas fornecem campo para trabalhos, inclusive teóricos, em campos que ainda estão por explorar adequadamente. Por exemplo, Foss e Foss (2004) apontam a integração da RBV com a TCT como uma evolução necessária para aquela, ou, Pandza et al. (2003) considerando a abordagem de real options como uma ferramenta adequada para a 
identificação de recursos para o desenvolvimento de capacidades futuras. Halawi, Aronson e Mc Carthy, (2005) discutem a RBV sob o ponto de vista da gestão do conhecimento.

Mahoney (2005), como foi mencionado, propõe algumas sugestões para pesquisas futuras que, em parte, coincidem com as agendas propostas por Carneiro et al.( 1999, ver Quadro 4) e Bandeira de Mello e Cunha (2001, ver Quadro 4): quais são e qual o papel das rotinas para a vantagem competitiva sustentável; a ligação de property rights, TCT e teoria da agência; top management team e recursos humanos influenciando a vantagem competitiva; a questão antiga, que ainda permanece, de como as firmas diferem em recursos, capacidades e desempenho econômico; formação e organização de redes e alianças para partilhar e desenvolver capacidades.

Esta agenda, afinal de contas, procura resolver as críticas a RBV que recaem em quatro argumentações: pelas definições imprecisas dos termos fundamentais; pelo seu teor tautológico redundante - por não identificar, a priori, se um determinado recurso é fundamental e qual a quantidade de recursos necessários, quer por se abster de determinar o valor dos recursos, focando a análise em recursos individuais - e, finalmente, pelo fato da RBV ser ou não uma novidade em função de seus antecedentes teóricos (WILLIAMSON, 1999; PRIEM; BUTLER, 2001; FOSS; KNUDSEN, 2003).

Barney (2001) vem em defesa da RBV argumentando que apesar de não existirem testes para identificar os recursos estratégicos, estes precisam ser avaliados nos contextos das firmas. Adicionalmente, se é verdade que a RBV não pode gerar uma lista de potenciais recursos, pode especificar características que estes devem possuir para serem fonte de vantagem competitiva. Diversos trabalhos procuraram avaliar o efeito de recursos e alguns em compreender melhor a sua natureza. Isto é, há alguma preocupação em determinar o que são e quais são os recursos estratégicos (ver ex. no Quadro 2). Entretanto, embora parte dos trabalhos selecionados tenha procurado compreender melhor os recursos intangíveis e constituam parte de projetos de pesquisas (ver observações acerca da Tabela 1), os demais parecem tratar-se de trabalhos isolados, eventualmente fruto da conveniência do acesso ao objeto de pesquisa. Não descartando a importância da identificação deste ou daquele recurso como importante, a compreensão de como "construir" os recursos intangíveis que geram vantagem competitiva parece ser um item importante para a agenda.

Um aspecto de fundamental importância e, apontado por Carneiro et al. (1999, ver Quadro 4) é o de viabilizar o uso da RBV no dia-a-dia das empresas. Este desafio parte do uso da RBV como suporte às pesquisas de conteúdo e formulação de estratégias, como foi feito por Baraniuk e Bulgacov (ver Quadro 2). No caso, não é criar uma lista ou uma idéia vaga de recursos fundamentais (por exemplo, capacidade de inovação), mas de compreender fatores ou características fundamentais para a avaliação, desdobramento e desenvolvimento de recursos.

Estes estudos tendem a ser "testados" em organizações brasileiras, porém, existem poucas organizações de grande porte no país. Os exemplos que merecem destaque são: CVRD, Petrobras, Gol e Gerdau. O sucesso comercial destas empresas tem efetivamente o potencial de inspirar a descoberta das melhores práticas, recursos e competências que poderão, eventualmente, ser "imitadas" por empresas seguidoras. Uma das sugestões para agendas futuras é estudar estes casos de sucessos de empresas brasileiras de porte.

Diversos trabalhos abordaram a fronteira da RBV com outras áreas como empreendedorismo (ROSÁRIO; BARBOSA, 2002, ver Quadro 3), recursos humanos (HOFMANN, 2002, ver Quadro 2), gestão do conhecimento(PIRES; MARCONDES, 2004, ver Quadro 2), uma tendência importante como apontado por Barney (1991).

Dois aspectos devem ser ressaltados: o primeiro diz respeito à necessidade de uniformização de conceitos que ainda perdura, conforme Carneiro et al. (1999) sugeriram, sobretudo, no que diz respeito a recursos e capacidade e/ou competências; o segundo pelo fato de poucos autores dedicaremse a projetos de pesquisa que considerem a abordagem de RBV, considerando as publicações dos EnANPAD, conforme foi apresentado.

Apesar de ter sido considerada uma abordagem emergente e importante para a explicação da vantagem competitiva nas organizações (RamosRodrigues; Ruiz-Navarro, 2004), os trabalhos de pesquisa em RBV no Brasil, considerando as 
publicações dos EnANPAD, não representam uma agenda de pesquisa estruturada. As exceções, em princípio, recaem sobre os autores apresentados e discutidos no Quadro 1. A existência de projetos e grupos de pesquisa em RBV além de possibilitar o crescimento consistente da contribuição na área, possibilita uma maior facilidade de cooperação entre pesquisadores e, com certeza, uma possibilidade maior de reconhecimento internacional da pesquisa realizada no Brasil.

\section{CONCLUSÕES}

Acreditamos que o trabalho tenha possibilitado algumas contribuições fundamentais como a resposta à questão apresentada na introdução, ao apresentar a evolução da pesquisa brasileira em RBV. Uma outra contribuição relevante é apresentar uma agenda para pesquisa, com base nas agendas propostas por pesquisadores internacionais de renome e que, por sinal, correspondem em parte a agendas já propostas em trabalhos apresentados no EnANPAD (CARNEIRO; CAVALCANTE; SILVA, 1999; BANDEIRA DE MELLO; CUNHA, 2001, ver Quadro 4). Pelo exposto, as agendas foram seguidas parcialmente e, aparentemente, sem um projeto de pesquisa que levasse as contribuições incrementais para a formação de um conjunto de resultados consistente em muitos casos. Neste sentido, uma primeira limitação deste trabalho e que fará parte de pesquisa futura é avaliar e sistematizar o conteúdo destes trabalhos selecionados.

Outras limitações devem-se a que, apesar da importância do EnANPAD, existem diversas fontes de publicação, principalmente revistas científicas. Porém, numa observação preliminar, poucos artigos haviam sido publicados sobre RBV nas principais revistas. No entanto, um trabalho que seguirá a este será a verificação de quais trabalhos que foram apresentados nos EnANPADs e publicados, visto que esta pesquisa se limitou a examinar somente os currículos Lattes dos autores que apresentaram trabalhos mais de uma vez.

Vale ressaltar a importância do trabalho para os autores, pois o mesmo visa orientar sua pesquisa futura. Outro aspecto positivo é que o uso de análises deste tipo e de pesquisa bibliométrica possibilita uma melhor compreensão dos campos a serem estudados e das referências fundamentais para basear a pesquisa.
Como sugestão, além de mais projetos de pesquisa com foco em RBV, o intercâmbio e cooperação entre pesquisadores brasileiros, ainda muito isolados e reclusos a suas instituições, e mesmo com pesquisadores estrangeiros pode ser uma ação importante para diminuir este efeito.

\section{REFERÊNCIAS}

ALMEIDA, A. L.; MUNIZ, R. M. A construção da reputação organizacional como recurso estratégico: o papel dos gestores e a percepção dos Stakeholders. In: ENCONTRO ANUAL DA ASSOCIAÇÃO NACIONAL DE PÓS-GRADUAÇÃO E PESQUISA EM ADMINISTRAÇÃO, 29., 2005, Brasília. Anais... Brasília: EnANPAD, 2005. p. 1-16.

ALMEIDA, A. L. C.; BERTUCCI, J. L. O.; BELO, J. S. A influência da comunicação na identificação dos empregados com a organização e sua valorização como recurso estratégico. In: ENCONTRO ANUAL DA ASSOCIAÇÃO NACIONAL DE PÓS-GRADUAÇÃ̆O E PESQUISA EM ADMINISTRAÇÃO, 30., 2006, Salvador. Anais... Salvador: EnANPAD, 2006. p. 1-16.

AMIT, R.; SCHOEMAKER, P. J. H. Strategic assets and organizational rents. Strategic Management Journal, ABI/INFORM Global, NY, 14, p. 22-46, 1993.

ANDRADE, A. A gestão de universidades sob a ótica da teoria de recursos e capacidades. In: ENCONTRO ANUAL DA ASSOCIAÇÃO NACIONAL DE PÓS-GRADUAÇÃO E PESQUISA EM ADMINISTRAÇÃO, 30., 2006, Salvador. Anais... Salvador: EnANPAD, 2006. p. 1-16.

BANDEIRA DE MELLO, R., CUNHA, C. A natureza e a dinâmica das capacidades organizacionais no contexto brasileiro: uma agenda para pesquisas sobre a vantagem competitiva das empresas brasileiras. In: ENCONTRO ANUAL DA ASSOCIAÇÃO NACIONAL DE PÓS-GRADUAÇÃO E PESQUISA EM ADMINISTRAÇÃO, 25., 2002, Salvador. Anais... Salvador: EnANPAD, 2001. p. 1-16. 
BARANIUK, J.; BULGACOV, S. Conteúdo e recursos estratégicos de empresas novas entrantes provedoras de serviços de comunicação de dados do Paraná. In: ENCONTRO ANUAL DA ASSOCIAÇÃO NACIONAL DE PÓS-GRADUAÇÃO E PESQUISA EM ADMINISTRAÇÃO, 26., 2002, Salvador. Anais... Salvador: EnANPAD, 2002. p. 1-16.

BARNEY, J. B. Organizational culture: can it be a source of sustained competitive advantage? Academy of Management Review, v. 11, n. 3, p. 656-665, 1999.

Firm resources and competitive advantage. Journal of Management, v. 17, p. 99-120, 1991.

Is the resource-based theory a useful perspective for strategic management research? Yes. Academy of Management Review, v. 26, n. 1, p. 41-56, 2001.

BIGNETTI, L.; PAIVA, E. Estudo das citações de autores de estratégia na produção acadêmica Brasileira. In: ENCONTRO ANUAL DA ASSOCIAÇÃO NACIONAL DE PÓS-GRADUAÇÃO E PESQUISA EM ADMINISTRAÇÃO, 25., Rio de Janeiro. Anais... Rio de Janeiro: EnANPAD, 2001. p. 112.

BINDER, M. Rede de recursos: um modelo desenvolvido a partir do caso Gol Linhas Aéreas. In: ENCONTRO ANUAL DA ASSOCIACÃO NACIONAL DE PÓS-GRADUAÇÃ̆O E PESQUISA EM ADMINISTRAÇÃO, 27., 2003, Atibaia. Anais... Atibaia: EnANPAD, 2003. p.1-16.

BOYD, B. K. CFO duality and firm performance: a aontingency model. Strategic Management Journal, v. 16, n. 3, p. 1-312, 1995.

BRITO, E. P. Z. et al. Reputação corporativa e desempenho: uma análise empírica no setor bancário. In: ENCONTRO ANUAL DA ASSOCIAÇÃO NACIONAL DE PÓS-GRADUAÇÃO E PESQUISA EM ADMINISTRAÇÃ̃O, 24., 2005, Brasília. Anais... Brasília: EnANPAD, 2005. p. 1-16.
CARNEIRO, J.; CAVALCANTI, M. A.; SILVA, J. Os determinantes da sustentabilidade da vantagem competitiva na visão resource-based. In: ENCONTRO ANUAL DA ASSOCIAÇÃO NACIONAL DE PÓS-GRADUAÇÃO E PESQUISA EM ADMINISTRAÇÃO, 23., 1999, Foz do Iguaçu. Anais... Foz do Iguaçu: EnANPAD, 1999. p. 1-16.

CASTANIAS, R. P.; HELFAT, C. E. Managerial resources and rents. Journal of Management, v. 17, n. 1, p. 155-171, 1991.

CAVES, R.; PORTER, M. From entry barriers to mobility barriers. Quarterly Journal of Economics, v. 91, n. 2, p. 241-261, 1977.

COASE, R. The nature of the firm. Economica, v. 4, p. 386-405, Nov. 1937.

DeGEUS, A. Planejamento como aprendizado. In: STARKEY, K. Como as organizações aprendem. São Paulo: Futura, 1997. p. 115-125.

Campus, 1998.

DIAS, A.; GONÇALVES, C.; COLETA, K. Determinantes estratégicos do desempenho de empresas em ambientes turbulentos. In: ENCONTRO ANUAL DA ASSOCIAÇÃO NACIONAL DE PÓS-GRADUAÇÃO E PESQUISA EM ADMINISTRAÇÃO, 28., 2004, Curitiba. Anais... Curitiba: EnANPAD, 2004. p. 1-16.

DIAS, A. Relações entre tamanho, diversificação, gestão de recursos, participação de mercado e desempenho organizacional de companhias brasileiras de capital aberto. In: ENCONTRO ANUAL DA ASSOCIAÇÃO NACIONAL DE PÓS-GRADUAÇÃO E PESQUISA EM ADMINISTRAÇÃO, 30., 2006, Salvador. Anais... Salvador: EnANPAD, 2006. p. 1-16.

DIERICKX, I.; COOL, K. Asset stock accumulation and sustainability of competitive advantage. Management Science, v. 35, n. 12, p. 1504-1511, 1989. 
DOLABELLA, R.; BITENCOURT, C. A consolidação das competências organizacionais na vitivinicultura brasileira: um estudo de caso da vinícola Miolo. In: ENCONTRO ANUAL DA ASSOCIAÇÃO NACIONAL DE PÓS-GRADUAÇÃO E PESQUISA EM ADMINISTRAÇ̃̃O, 30., 2006, Salvador. Anais... Salvador: EnANPAD, 2006. p. 1-16.

FERREIRA, P.; MARIANO, S. Estratégia competitiva no mundo virtual: o caso Americanas.com. In: ENCONTRO ANUAL DA ASSOCIACÃO NACIONAL DE PÓSGRADUAÇÃO E PESQUISA EM ADMINISTRAÇÃO, 25., 2002, Salvador. Anais... Salvador: EnANPAD, 2002. p. 1-16.

FENSTERSEIFER, J.; WILK, E. Visão da firma baseada em recursos, clusters e performance: um estudo no setor vitivinícula do RS. In: ENCONTRO ANUAL DA ASSOCIAÇÃO NACIONAL DE PÓS-GRADUAÇÃO E PESQUISA EM ADMINISTRAÇÃO, 29., Brasília. Anais... Brasília: EnANPAD, 2005. p. 1-16.

FOSS, N. Research in strategy, economics and Michael Porter. Journal of Management Studies, v. 33, n. 1, p. 1-24, Jan. 1996.

FOSS, N.; KNUDSEN, T. The resource-based tangle. Search of Sustainable Foundations Managerial and Decision Economics, v. 33, n. 24, p. 291-307, 2003.

FOSS, K.; FOSS, N. The next step in the evolution of the RBV: integration with transaction costs economics. Copenhagen Business School, v. 3, n. 2, p. 75-86, 2004.

GHEMAWAT, P. Sustainable advantage. Harvard Business Review, n. 64, p. 53-58, 1986.

GRANOVETTER, M. Economic action and social structure: the problem of embeddedness.

American Journal of Sociology, v. 91, n. 3, p. 481-510, 1985.

HALAWI, L.; ARONSON, J.; McCARTHY, R. Resource-based view of knowledge management for competitive advantage. Journal of Knowledge, v. 7, p. 137-158, 2005.
HAMEL, G.; PRAHALAD, C. K. Competing for the future. Boston: Harvard Business School, 1994.

HEXSEL, A. O entendimento do sucesso internacional da Marcopolo: uma visão com base em recursos. In: ENCONTRO ANUAL DA ASSOCIAÇ̃̃O NACIONAL DE PÓSGRADUAÇÃO E PESQUISA EM ADMINISTRAÇÃO, 27., 2003, Atibaia. Anais... Atibaia: EnANPAD, 2003. p. 1-16.

HOFMANN, V. La ventaja competitiva basada em la estratégia de perfeccionamiento operacional de los recursos humanos: el caso de uma red de supermercados em Brasil. In: ENCONTRO ANUAL DA ASSOCIAÇÃO NACIONAL DE PÓS-GRADUAÇ̃̃O E PESQUISA EM ADMINISTRAÇÃO, 25., 2002, Salvador. Anais... Salvador: EnANPAD, 2002. p. 1-16.

HOOPES, D. G.; MADSEN, T. L.; WALKER, G. Guest editors' introduction to the special issue: why is there a resource-based view? toward a theory of competitive heterogeneity. Strategic Management Journal, v. 24, p. 889-902, 2003.

JACQUES, J. A importância da análise do contexto organizacional durante a construção e implementação de recursos assistenciais na gestão da criação do conhecimento: uma contribuição para o processo estratégico nas organizações hospitalares. In: ENCONTRO ANUAL DA ASSOCIAÇÃO NACIONAL DE PÓS-GRADUAÇÃO E PESQUISA EM ADMINISTRAÇÃO, 30., 2006, Salvador. Anais... Salvador: EnANPAD, 2006. p. 1-16.

JOYCE, W. et al. O que (realmente) funciona: as melhores práticas das empresas de sucesso. Rio de Janeiro: Campus, 2003.

KAY, J. Foundations of corporate success. Oxford: Oxford University, 1993.

KAY, J. et al. The history of strategy and some thoughts about the future. In: FAULKNER, D.; CAMPBELL, A. (Org.). Oxford handbook of strategy: a strategy overview and competitive strategy. Oxford: Oxford University, 2003. v. 1. 
KAYO, E.; BASSO, F.; PENNER-HAHN, J. The value relevance of intangible capabilities deployment: the role of firm life cycle. In: ENCONTRO ANUAL DA ASSOCIAÇÃO NACIONAL DE PÓS-GRADUAÇÃO E PESQUISA EM ADMINISTRAÇÃO, 30., 2006, Salvador. Anais... Salvador: EnANPAD, 2006. p. 1-16.

KIMURA, H.; MARTIN, D.; NAKAMURA, W. Modelagem de economic rents através das vantagens de capability-building e de resourcepicking. In: ENCONTRO ANUAL DA ASSOCIACAAO NACIONAL DE PÓS-GRADUAÇÃO E PESQUISA EM ADMINISTRAÇÃO, 28., 2004, Curitiba. Anais... Curitiba: EnANPAD, 2004. p. 1-16.

KOHLS, V.; FENSTERSEIFER, J. Estratégias competitivas de empresas agroalimentares: estudos de casos na zona sul do RS. In: ENCONTRO ANUAL DA ASSOCIAÇ̃̃O NACIONAL DE PÓS-GRADUAÇ̃̃O E PESQUISA EM ADMINISTRAÇÃO, 28., 2004, Curitiba. Anais... Curitiba, EnANPAD, 2004. p. 1-16.

LEVITT, B.; MARCH, J. Organizational learning. Annual Review of Sociology, v. 14, p. 319-340, 1988.

LEWIN, A. et al. The coevolution of new organizational forms. Organization Science, v. 10 , n. 5 , p. 535-550, 1999.

LIPPMAN, S. A.; RUMELT, R. P. Uncertain imitability. Bell Journal of Economics, v. 13, n. 2, p. 418-438, 1982.

MAHONEY, J. T.; PANDIAN, J. R. The resource-based view within the conversation of strategic management. Strategic Management Journal, v. 13, n. 5, p. 363-380, 1992.

MAHONEY, J. T. The management of resources and the resource of management. Journal of Business Research, v. 33, n. 2, p. 91-101, 1995.

A resource-based theory of sustainable rents. Journal of Management, v. 27, n. 6, p. 651-660, 2001.
Economic foundations of strategy. Thousand Oaks, CA: Sage, 2005.

MEIRELLES, D.; BASSO, L.; PACE, E. Um estudo dos relacionamentos externos da empresa: Uma aplicação da teoria baseada em recursos ao setor de Software. In: ENCONTRO ANUAL DA ASSOCIAÇÃO NACIONAL DE PÓS-GRADUAÇÃO E PESQUISA EM ADMINISTRAÇÃO, 29., 2005, Brasília. Anais... Brasília: EnANPAD, 2005. p.1-16.

NELSON, R.; WINTER, S. An evolutionary theory of economic change. London: Belknap Press, 1982.

OMAKI, E. Recursos intangíveis e desempenho em grandes empresas brasileiras: avaliações dos recursos intangíveis como estimadores de medidas de desempenho financeiras. In: ENCONTRO ANUAL DA ASSOCIAÇÃO NACIONAL DE PÓS-GRADUACÃ̃ E PESQUISA EM ADMINISTRAÇÃO, 29., 2005, Brasília. Anais... Brasília, EnANPAD, 2005. p. 1-16.

PANDZA, K. et al. A real options approach to managing resources and capabilities. International Journal of Operations \& Production Management, v. 23, n. 9, 2003, p. 1010-1032.

PASCUCCI, L.; CRUBELLATE, J.; GRAVE, P. Ambiente institucional, recursos e comportamento estratégico: o estudo da verticalização de negócios em uma Unidade Regional da UNIMED. In: ENCONTRO ANUAL DA ASSOCIAÇÃO NACIONAL DE PÓS-GRADUAÇÃO E PESQUISA EM ADMINISTRAÇÃO, 30., 2006, Salvador. Anais... Salvador: EnANPAD, 2006. p. 1-16.

PASCUCCI, L.; GRAVE, P.; SILVEIRA, J. A UNIMED Maringá e seu posicionamento estratégico: uma explicação a partir da visão baseada em recursos. In: ENCONTRO ANUAL DA ASSOCIAÇÃO NACIONAL DE PÓSGRADUAÇ̃̃O E PESQUISA EM ADMINISTRAÇÃO, 27., 2003, Atibaia. Anais... Atibaia: EnANPAD, 2003. 
PENROSE, E. The theory of the growth of the firm. Oxford: Oxford University, 1959.

PEREIRA, M.; FORTE, S. Visão baseada em recursos nas instituições de ensino superior de Fortaleza: uma análise ex-ante e ex-post à LDB/96. In: ENCONTRO ANUAL DA ASSOCIAÇÃO NACIONAL DE PÓS-GRADUAÇÃO E PESQUISA EM ADMINISTRAÇĂ̊O, 28., 2004, Curitiba. Anais... Curitiba: EnANPAD, 2004.

PETERAF, M. A. The cornerstones of competitive advantage: a resource-based view. Strategic Management Journal, v. 14, p. 179191, 1993.

PIRES, M.; MARCONDES, R. A vantagem competitiva nas organizações financeiras: uma análise baseada na teoria dos recursos. In: ENCONTRO ANUAL DA ASSOCIAÇÃO NACIONAL DE PÓS-GRADUAÇÃO E PESQUISA EM ADMINISTRAÇÃO, 28., 2004, Curitiba. Anais...Curitiba: EnANPAD, 2004. p. 1-16.

PORTER, M. E. Competitive strategy: techniques for analyzing industries. New York: Free, 1980.

PRAHALAD, C. K.; HAMEL, G. The core competence of the organization. Harvard Business Review, v. 3, n. 2, p. 79-91, May/ June 1990.

PRIEM, R.; BUTLER, J. Tautology in the resource-based view and the implications of externally determined resource value: further comments. Academy of Management Review, v. 26, n. 1, p. 57-66, 2001.

PROENÇA, A. Dinâmica estratégica sob uma perspectiva analítica: refinando o entendimento gerencial. Arché Interdisciplinar, v. 23, p. 95-133, 1999.

RAMOS-RODRIGUEZ, A. R.; RUIZNAVARRO, J. Changes in the intellectual structure of strategic management research: a bibliometric study of the Strategic Management Journal, 1980- 2000. Strategic Management Journal, v. 25, p. 981-1004, 2004.
ROSÁRIO, F. J. P.; BARBOSA, J. D. Recursos e posicionamento competitivo nas PME'S. In: ENCONTRO ANUAL DA ASSOCIAÇÃO NACIONAL DE PÓS-GRADUAÇÃ O E PESQUISA EM ADMINISTRAÇÃO, 23., 2002, Salvador. Anais... Salvador: EnANPAD, 2002. p. 14.

ROSS, S. The economic theory of agency: the principal's problem. American Economic Review, v. 63, p. 134-139, 1973.

RUMELT, R. P. Towards a strategic theory of the firm. In: LAMB, R. B. (Ed.). Competitive strategic management. Upper Sadler River: Prentice Hall, 1984. p. 95-117.

RUMELT, R. P. Theory, strategy and entrepreneurship. In: TEECE, D. (Ed.). The competitive challenge: strategies for industrial innovation and renewal. Cambridge: Ballinger, 1987. Chapter 7, p. 137-158.

SERRA, F. et al. A Administração estratégica: conceitos, roteiro prático e casos. Rio de Janeiro: Reichmann \& Affonso, 2002.

SILVA, J.; OLIVEIRA, C. Grupos estratégicos e a visão resource-based view: uma aplicação de sistemas Neuro-fuzzy. In: ENCONTRO ANUAL DA ASSOCIAC ÃO NACIONAL DE PÓS-GRADUAÇÃO E PESQUISA EM ADMINISTRAÇÃO, 29., 2005, Brasília. Anais... Brasília: EnANPAD, 2005.

SOUSA, J.; FERREIRA, F.; MOURA, S. Proposta de categorização dos recursos estratégicos de IES particular: uma contribuição com base nos modelos da VBR e da hiper-competição. In: ENCONTRO ANUAL DA ASSOCIACÃO NACIONAL DE PÓS-GRADUAÇĂ̊ E PESQUISA EM ADMINISTRAÇÃO, 24., 2005, Brasília. Anais... Brasília: EnANPAD, 2005.

THOMAZ, J. C.; LERNER, E. B.; BRITO, E. P. Reputação corporativa: desenvolvendo uma escala de mensuração. In: ENCONTRO ANUAL DA ASSOCIAÇÃO NACIONAL DE PÓS-GRADUAÇÃO E PESQUISA EM ADMINISTRAÇÃO, 30., 2006, Salvador. Anais... Salvador: EnANPAD, 2006. 
VERGARA, S. C. Projetos e relatórios de pesquisa em administração. São Paulo: Atlas, 1997.

WERNERFELT, B. A resource-based view of the firm. Strategic Management Journal, v. 5 , p. $171-180,1984$.

WILLIAMSON, O. The economic institutions of capitalism: firms, markets, relational contracting. New York: Free, 1985.

WILLIAMSON, O. Strategy research: governance and competence perspectives. Strategic Management Journal, v. 20, n. 12, p. 1087-1108, 1999.

WILK, E.; FENSTERSEIFER, J. Alianças estratégicas sob a perspectiva da visão da firma baseada em recursos: Contribuições para um modelo dinâmico de cooperação. In: ENCONTRO ANUAL DA ASSOCIAÇÃO NACIONAL DE PÓS-GRADUAÇÃO E PESQUISA EM ADMINISTRAÇÃO, 27., 2003, Atibaia. Anais... Atibaia: EnANPAD, 2003.

Strategy research: governance and competence perspectives. Strategic Management Journal, v. 20, n. 12, p. $1087-$ 1108, 2000.

ZUCKER, L. Institutional theories of organization. Annual Review of Sociology, v. 13, p. 443-464, 1987.

Recebido: 29/07/2007 Received: 07/29/2007

Aprovado: 08/08/2007 Approved: 08/08/2007 